\title{
A importância da monitorização intraoperatória multimodal nas cirurgias espinais
}

\author{
Margareth Reiko Kai', Cristiano Augusto Cruz e Silva², Joseph Bruno \\ Bidin Brooks², Ricardo Botelho ${ }^{3}$, José Marcus Rotta ${ }^{4}$
}

Setor de Eletrodiagnóstico do Hospital do Servidor Público Estadual de São Paulo

Serviço de Neurocirurgia do Hospital do Servidor Público Estadual de São Paulo

\section{RESUMO}

A monitorização intraoperatória multimodal (MIOM) tornou-se um método amplamente utilizado para monitorização da função neural durante as cirurgias espinais. Um estudo prospectivo de 52 pacientes que foram monitorados durante os procedimentos cirúrgicos, entre outubro de 2009 e dezembro de 2010, no Hospital do Servidor Público Estadual de São Paulo, a fim de determinar a sensibilidade e especificidade das técnicas utilizadas para monitorar a medula espinhal e raízes nervosas durante as cirurgias espinais. Portanto, o objetivo deste estudo prospectivo foi avaliar a eficácia da MIOM, que inclui os potenciais evocados somatossensitivos (PESS), os potenciais evocados motores com estimulação transcraniana (MEPS), a eletromiografia contínua (EMG) captada nos músculos eletricamente estimulados a partir do córtex motor, na própria medula espinal, na cauda equina e nas raízes nervosas, comparando os dados eletrofisiológicos com os achados clínicos neurológicos no pós-operatório imediato. A MIOM visa monitorar tanto as vias ascendentes quanto as descendentes, fornecendo informações com feedback imediato sobre qualquer déficit neurológico durante o procedimento que permita uma identificação rápida de possíveis danos das estruturas neurais e prevenção por meio de ações corretivas. Técnicas que visam apenas avaliar as vias ascendentes ou as vias descendentes isoladamente não podem fornecer resultados com sensitividade ou especificidade adequados. Cinquenta e dois pacientes foram submetidos à MIOM. Desses, 49 casos foram verdadeiramente negativos, dois casos foram verdadeiramente positivos e 1 caso foi falso-positivo, resultando em sensibilidade de $100 \%$ e especificidade de $98 \%$ ( $p$ >0,05). Não foram obtidos falsos-negativos. Com base nos resultados deste estudo, a MIOM é um método eficaz de monitorar a integridade funcional da medula espinal e, consequentemente, melhorar os resultados pós-operatórios.

\section{PALAVRAS-CHAVE}

Monitorização intraoperatória, potenciais evocados, cirurgia espinal.

\section{ABSTRACT}

The value of multimodal intraoperative monitoring during spinal surgery

Multimodal intraoperative monitoring (MIOM) has become a widely used method of monitoring neural function during spine surgery. A prospective study of 52 patients who received MIOM during spine surgery procedures between October 2009 and December 2010 at Hospital do Servidor Público Estadual de São Paulo, in order to determine the sensitivity and specificity of MIOM techniques used to monitor spinal cord and nerve roots function during spine surgery. Therefore, the purpose of this prospective study was to evaluate the efficacy of somatosensory evoked potentials (SSEP), transcranial motor evoked potentials (MEP), and continuous electromyography (EMG) recorded from the spinal cord and muscles electrically elicited from the motor cortex, spinal cord, cauda equina and nerve root stimulation and compare the neurophysiological data with immediate post-operative clinical neurological outcome. MIOM aims to monitor both ascending and descending pathways, giving immediate feedback information regarding any neurological deficits during the operation allowing rapid identification of potential damage of the neural structures and avoidance through corrective action. Techniques that only evaluate either ascending or descending pathways, may not provide sensitive or specific results. Fifty two patients underwent MIOM to evaluate any neural deficits that may have occurred during spine surgery. Of these, 49 were true negative cases, 2 were true positive cases and 1 was false positive case, resulting in a sensitivity of $100 \%$ and a specificity of $98 \%$ $(p>0.05)$. None were false negative. Based on the results of this study, MIOM is an effective method of monitoring the spinal cord functional integrity and consequently improve postoperative results.

\section{KEYWORDS}

Intraoperative monitoring, evoked potentials, spine surgery.

1. Chefe do Setor de Eletrodiagnóstico do Hospital do Servidor Estadual de São Paulo.

2. Médico estagiário do Setor de Eletrodiagnóstico do Hospital do Servidor Estadual de São Paulo.

3. Neurocirurgião do Hospital do Servidor Estadual de São Paulo.

4. Chefe do Serviço de Neurocirurgia do Hospital do Servidor Estadual de São Paulo. 


\section{Introdução}

No início dos anos 1970, com o advento de técnicas cirúrgicas mais agressivas, a instrumentação espinhal começou a ser mais utilizada para o tratamento de graves deformidades da coluna vertebral, levando ao desenvolvimento da monitorização intraoperatória da medula espinal. As complicações neurológicas das cirurgias espinais com ou sem instrumentação variam de $1 \%$ a 33\% ${ }^{1,2,12,13,19}$ A paraplegia ou a tetraplegia iatrogênica é um resultado devastador para o paciente e para o cirurgião, que pode ocorrer apesar de todos os cuidados.

Na última década tem havido evolução significativa da neurofisiologia intraoperatória. Isso é particularmente verdadeiro para os métodos de controle dos tratos motores durante cirurgias da coluna vertebral e da medula espinal. Vários autores concordam que o uso de potenciais evocados somatossensitivos (PESS) isoladamente apenas para monitorar a coluna dorsal não é confiável para detectar ou evitar a lesão do trato corticoespinal. Após anos de experiências, tornou-se cada vez mais evidente que uma combinação dos potenciais evocados motores (MEPs) produz melhores resultados para a prevenção e a detecção das lesões neurológicas da medula espinal, seja por ação direta da cirurgia ou indiretamente induzida, por exemplo, por alterações hemodinâmicas. ${ }^{4}$ Portanto, a combinação da estimulação transcraniana com estímulos elétricos em multipulsos do córtex motor com o registro das respostas motoras nos músculos, associada aos PESS, é a melhor forma de avaliar as vias ascendentes e descendentes da medula espinal.

A eletromiografia contínua demonstra em tempo real a irritação da raiz nervosa, permitindo a intervenção e a minimização dos riscos de lesão. ${ }^{2}$

O objetivo do presente estudo foi determinar a sensitividade e a especificidade das técnicas utilizadas na monitorização intraoperatória multimodal nas cirurgias espinais.

\section{Material e métodos}

Utilizou-se o equipamento Endeavor CR, Viasys Healthcare, 16 canais, com estimuladores integrados. Eletrodos de superfície foram utilizados para a estimulação dos nervos periféricos para obtenção dos potenciais evocados somatossensitivos (PESS). Eletrodos subdérmicos monopolares foram utilizados para a captação dos registros dos MEPs nos músculos determinados, de acordo com a programação cirúrgica, assim como para a estimulação transcraniana motora e a captação dos registros corticais dos potenciais evocados soma- tossensitivos posicionados em C3', C4' e CZ, de acordo com o sistema internacional 10-20 para EEG. $^{3}$

As estruturas neurais a serem estimuladas e os locais de registro eram determinados de acordo com o procedimento cirúrgico. A monitorização foi sempre realizada em ambos os lados do corpo, com registro simultâneo. Sempre foram monitorizados níveis proximais e distais à lesão, para distinguir alterações sistêmicas (hipotermia, hipotensão, anestesia etc.) das alterações relacionadas ao procedimento cirúrgico. Os métodos utilizados foram: potenciais evocados somatossensitivos (PESS), potenciais evocados motores (MEPs), eletromiografia contínua, eletromiografia estimulada e eletroencefalograma contínuo (EEG).

Em todos os casos foi usada anestesia totalmente intravenosa, usualmente com propofol e fentanil. ${ }^{14}$ Relaxante muscular foi utilizado somente para a entubação, exceto nos casos em que havia necessidade de monitorização durante o posicionamento do paciente. Foram monitorizados 52 pacientes, sendo 22 do sexo feminino e 30 do sexo masculino, com idade média de 59,3 anos (variando de 6 a 89 anos).

\section{Resultados}

O primeiro registro de todas as modalidades é realizado com o paciente já anestesiado e é considerado a nossa linha de base, que é armazenada no computador. Essa linha de base é o nosso parâmetro para avaliar as alterações que poderão ocorrer durante o procedimento.

Prolongamento das latências maior que $10 \%$ e queda das amplitudes maior que $50 \%$ em relação à linha de base inicial foram considerados sinais de alarme., ${ }^{4,14,15}$

Dos 52 pacientes, 49 casos foram verdadeiramente negativos, 2 casos foram verdadeiramente positivos (Tabela 1) e 1 caso foi falso-positivo, resultando em uma sensibilidade de $100 \%$ e uma especificidade de $98 \%$ $(p>0,05)$. Não foram obtidos falsos-negativos.

\section{Discussão}

Na década de 1970, com os avanços tecnológicos dos métodos diagnósticos e cirúrgicos das patologias espinais, cada vez mais invasiva, houve uma preocupação com a proteção da medula espinal, e nesse ambiente se iniciou o desenvolvimento da monitorização intraoperatória. Tamaki e cols. desenvolveram os potenciais evocados espinais, com estimulação e registro na medula espinal. ${ }^{6,16-18}$ 


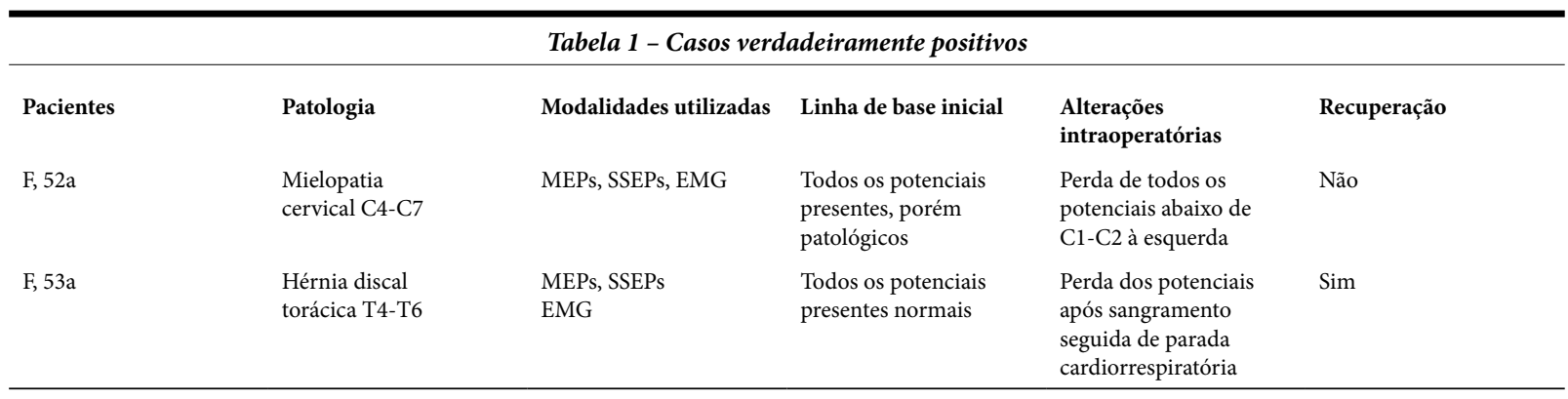

Nash e cols. implementaram os potenciais evocados somatossensitivos durante o procedimento cirúrgico. ${ }^{10,11}$ Entretanto, todos esses métodos avaliavam somente a coluna dorsal da medula. $\mathrm{O}$ único método para avaliar a parte motora era o wake-up test, o teste do despertar desenvolvido por Vauzelle e cols. ${ }^{20}$

Na década de 1980, ocorreu o grande avanço da monitorização, quando Merton e Morton relataram a técnica de estimulação transcraniana do córtex motor. ${ }^{9}$ Vários autores já observavam que os potenciais evocados somatossensitivos apresentavam resultados falsos-positivos como Lesser e cols., em 1986. ${ }^{7}$

Nos últimos 15 anos, a monitorização intraoperatória se tornou um método mais frequentemente utilizado e, com a experiência adquirida, chegamos na era da monitorização intraoperatória multimodal, na qual várias técnicas são utilizadas em conjunto - PESS, MEPS, EMG contínua e estimulada e EEG. Técnicas utilizadas isoladamente devem ser evitadas, com o risco de não abranger a complexa função da medula espinal.

As estruturas neurais a serem estimuladas e os locais de registro devem ser escolhidos caso a caso. A seleção dos músculos com a maior representação do trato corticoespinal e da quantidade a ser utilizada para o registro dos MEPs é extremamente importante, pois em pacientes com comprometimento motor importante no pré-operatório é possível que não se obtenham os MEPs no intraoperatório. A monitorização deve ser realizada bilateralmente, e sempre nos membros superiores e inferiores, independentemente do local da lesão, para distinguir alterações sistêmicas devidas a anestesia, temperatura ou acidose, por exemplo, das alterações diretamente relacionadas com o procedimento cirúrgico, além da preservação do plexo braquial e dos nervos periféricos decorrente da posição do paciente durante a cirurgia. ${ }^{4,5}$ Por isso, a importância de um equipamento com um grande número de canais e estimuladores acoplados.

O objetivo deste trabalho foi a avaliação da sensitividade e especificidade do método nas cirurgias espinais no nosso serviço, englobando várias patologias.

Para aumentar a precisão diagnóstica da monitorização intraoperatória, as configurações foram "personalizadas" para cada paciente. Cada caso foi discutido com a equipe cirúrgica, sendo considerados a patologia, as estruturas neurais em risco e o exame neurológico antes da cirurgia. Esses dados são cruciais para a correta interpretação e análise dos registros durante o procedimento cirúrgico, para que se possa alertar o cirurgião quando alterações verdadeiramente positivas ocorrerem indicando um comprometimento das estruturas neurais. Caso ocorram, será possível prevenir lesões permanentes com manobras corretivas, ajustes da hemodinâmica do paciente ou utilização de metilpredinisolona e, em casos extremos, interrupção do procedimento.

Sutter e cols. denominaram casos de falso-positivo de "distúrbios desnecessários" do procedimento cirúrgico pelo neurofisiologista. ${ }^{14} \mathrm{E}$ talvez realmente tenha sido o que ocorreu no nosso caso. Considerou-se um prolongamento assimétrico das latências dos potenciais somatossensitivos nos membros inferiores em um caso de mielopatia cervical, sem outras alterações associadas, seja hemodinâmica ou motora.

Nossos resultados confirmam a alta sensitividade e especifidade do método descrito na literatura e justificam que um neurofisiologista com experiência deveria sempre fazer parte da equipe cirúrgica quando estruturas neurais estejam em risco.

Nossa experiência também confirma que a integração entre cirurgião, neurofisiologista e anestesista é a chave para uma monitorização bem-sucedida., ${ }^{714}$

\section{Conclusão}

Os resultados obtidos neste estudo comprovam a alta especificidade e sensitividade do método. $\mathrm{O}$ uso combinado das técnicas PESS e MEPs deveria ser implementado em todos os centros que realizam cirurgias espinais, sempre com a monitorização realizada de forma multimodal, para detectar e prevenir lesões da medula espinal. Técnicas de monitorização tais como PESS ou eletromiografia contínua, realizadas isoladamente, devem ser evitadas, pois não são suficientes para avaliar a complexa função das vias ascendentes e descendentes da medula espinal e das raízes nervosas. 


\section{Referências}

1. Agazzi S, Reverdin A, May D. Posterior lumbar interbody fusion with cages: an independent review of 71 cases. J Neurosurg. 1999;91:186-92.

2. Elias WJ, Simmons NE, Kaptain GJ, Chadduck JB, Whitehill R. Complications of posterior lumbar interbody fusion when using a titanium threaded cage device. J Neurosurg. 2000;93:45-52.

3. Bose B, Wierzbowski LR, Sestokas AK. Neurophysiologic monitoring of spinal nerve root function during instrumented posterior lumbar spine surgery. Spine. 2002;27:1444-50.

4. Deletis V, Sala F. Intraoperative neurophysiological monitoring during spine surgery: an update. Curr Opin Orthop. 2004;15:154-8.

5. Deletis V. Intraoperative neurophysiology and methodology used to monitor the functional integrity of the motor system. In: Deletis V, editor. Neurophysiology in neurosurgery: a modern intraoperative approach. Dublin: Academic; 2002. p. 25-51.

6. Kurokawa T. Spinal cord action potentials evoked by epidural stimulation of the spinal cord: a report of human and animal record [in Japanese]. Nouha to Kindennzu (Jpn J Electroenceph Electromyogr). 1972;1:64-6.

7. Lesser RP, Raudzens P, Luders H, Nuwer MR, Goldie WD, Morris $\mathrm{HH}$, et al. Postoperative neurological deficits may occur despite unchanged intraoperative somatosensory evoked potentials. Ann Neurol. 1986;19:22-5.

8. Macdonald DB, Al Zayed Z. Four-limb muscle motor evoked potential and optimized somatosensory evoked potential monitoring with decussation assessment: results in 206 thoracolumbar spine surgeries. Eur Spine J. 2007(Suppl) 2:S171-87.

9. Merton PA, Morton HB. Stimulation of the cerebral cortex in the intact human subject. Nature. 1980;285:227.

10. Nash CL, Brodkey JS, Croft TJ. A model for electrical monitoring of spinal cord function in scoliosis patients undergoing correction [abstract]. J Bone Joint Surg (Am). 1972;54:197-8.

11. Nash CL, Lorig RA, Schatzinger LA, Brown RH. Spinal cord monitoring during operative treatment of the spine. Clin Orthop Rel Res. 1977;12:100-5.

12. Okuyama K, Abe E, Suzuki T, Tamura Y, Chiba M, Sato K. Posterior lumbar interbody fusion: a retrospective study of complications after facet joint excision and pedicle screw fixation in 148 cases. Acta Orthop Scand. 1999;70:329-34.

13. Pihlajamaki H, Myllynen P, Bostman O. Complications of transpedicular lumbosacral fixation for non-traumatic disorders. J Bone Joint Surg Br. 1997;79:183-9.

14. Sutter M, Eggspuhler A, Grob D, Jeszenszky D, Porchet $\mathrm{F}$, Muller A, et al. The diagnostic value of multimodal intraoperative monitoring (MIOM) during spine surgery: a prospective study of 1017 cases. Eur Spine J. 2007;16(Suppl 2):S162-70.

15. Szelenyi A, Kothbauer K, Deletis V. Transcranial electric stimulation for intraoperative motor evoked potential monitoring: stimulation parameters and electrode montages. Clin Neurophysiol. 2007;118:1586-95. [doi: 10.1016/j.clinph.2007.04.008].

16. Tamaki T, Kobayashi H, Yamane T, Egashira T, Tsuji H, Inoue S. Clinical application of evoked spinal cord evoked potential [in Japanese]. Seikeigeka. Orthopaedic Surgery. 1977;28:681-9.

17. Tamaki T, Yamane T. Proceedings: clinical utilization of the evoked spinal cord action potential in spine and spinal cord surgery (abstract). Electroencephalogr Clin Neurophysiol. 1975;39:539.

18. Tamaki T, Yamashita T, Kobayashi H, Hiryama H. Spinal cord evoked potential after stimulation to the spinal cord (SCEP). Spinal cord monitoring basic data obtained from animal experimental studies [abstract in Japanese]. Nouha to Kindennzu (Jpn J Electroenceph Electromyogr). 1972;1:196.

19. Thomsen K, Christensen FB, Eiskjaer SP, Hansen ES, Fruensgaard S, Bunger CE. 1997 Volvo Award winner in clinical studies: the effect of pedicle screw instrumentation on functional outcome and fusion rates in posterolateral lumbar spinal fusion: a prospective, randomized clinical study. Spine. 1997;22:2813-22.

20. Vauzelle C, Stagnara P, Jouvinroux P. Functional monitoring of spinal cord activity during spinal surgery. Clin Orthop. 1973;93:173-8.

Endereço para correspondência

Margareth Reiko Kai

Rua Dr. Pinto Ferraz, 97, ap. 102, Vila Mariana

04117-040 - São Paulo, SP

E-mail: m.reiko@hotmail.com

Telefones: (11) 5088-8281/5575-4368. Celular: (11) 8145-4780 